\title{
Les besoins de santé des femmes séropositives ayant recours à une association telle que Aides
}

\author{
Anne-Déborah BouHNIK ${ }^{1}$, Graciela CATTANEO et Jean-Marie LE GALL ${ }^{2}$
}

\begin{abstract}
Résumé
L'analyse des données de l'enquête « Aides et toi - 2005 » effectuée sur un échantillon de 1932 personnes montre que la moitié des femmes répondantes sont infectées par le VIH/sida et que $38 \%$ de ces femmes séropositives sont des immigrées, essentiellement d'Afrique sub-saharienne.

Ces femmes cumulent des éléments défavorables pour ce qui concerne leur qualité de vie, les soins et la prévention, facteurs qui tiennent à leurs conditions de vie : isolement, grande précarité sociale, économique et relationnelle. Ils sont plus marqués pour les femmes accueillies à Aides que pour la population générale des femmes séropositives suivies à l'hôpital et étudiées par l'enquête Anrs-Vespa.

Les femmes immigrées/étrangères sont plus fragilisées économiquement et socialement. Elles ressentent davantage de discriminations, notamment intracommunautaires.

Les actions d'une association telle que Aides, par les actions qu'elle mène, constitue un lieu d'expression des besoins de santé des femmes les plus précarisées, notamment pour les immigrées. L'identification de ces difficultés appuie l'élaboration de réponses collectives en termes de soutien.
\end{abstract}

Mots clés : femmes, VIH, immigrées, précarité, isolement, discrimination, santé, communautaire.

En France, peu de données sont disponibles sur les femmes en contact avec les associations de lutte contre le sida. L'existence de ces associations constitue cependant une des composantes sociales importantes pour ce qui concerne le sida, permettant aux personnes atteintes d'obtenir des informations, du soutien et de participer à un mouvement collectif de lutte pour l'amélioration de leurs conditions de vie.

"Aides et toi » est une enquête nationale, réalisée dans le cadre d'une convention entre l'association Aides et la Direction générale de la santé (DGS). Nous faisons référence ici à l'étude réalisée du 9 au 15 mai 2005 [1] et proposée à toutes les personnes qui fréquentent les accueils de Aides ou qui participent aux actions régulières menées par cette association. Cette enquête permet d'approcher toutes les populations rencontrées à Aides et montre que les femmes représentent un tiers des personnes accueillies ; par ailleurs, les femmes constituent un tiers des personnes séropositives et un tiers de la file active (sujets soignés

\footnotetext{
Observatoire de la santé de la région PACA/unité Inserm 379, Marseille.
} Aides, Pantin. pour le $\mathrm{VIH} / \mathrm{sida}$ et régulièrement suivis) des personnes séronégatives accueillies. Les données 2005 sur la répartition respective (en pourcentage) des femmes dans ces sous-catégories ne présentent pas d'évolution par rapport aux données de nos enquêtes de 2002 et $2004^{3}$, et confirment le fait que les femmes et le réseau de prise en charge médico-psychosocial ont identifié Aides comme un lieu ressource pour les femmes séropositives.

Nous constatons également qu'une femme sur deux accueillies à Aides est séropositive, proportion identique à chaque vague d'enquête.

Ces données doivent être mises en perspective avec le chiffre de la file active des personnes annuellement rencontrées par Aides, qui est estimé entre 27000 et 30000 personnes [2]. Nous verrons que le mode de recrutement de cette enquête fait émerger des catégories de femmes parfois peu représentées dans celles habituellement menées auprès des personnes séropositives, très largement recrutées en milieu hospitalier.

L'objectif de cet article est de décrire la population desfemmes séropositives ayant répondu à cette enquête. La comparaison avec les données de l'enquête Anrs-Vespa [3], représentative des personnes vivant avec le VIH en France, nous permettra d'évaluer les spécificités des femmes séropositives fréquentant l'association, en termes de caractéristiques sociodémographiques, par rapport aux autres femmes infectées par le VIH/sida recrutées dans une file active hospitalière. Les données portant sur la sexualité nous permettront également de comparer leurs comportements de prévention.

Par ailleurs, l'analyse, basée sur les trois vagues d'enquête, approfondira les raisons déclarées de la démarche de ces femmes vers Aides, ainsi que les questions relatives notamment aux discriminations qu'elles subissent, sujet peu documenté dans les autres enquêtes portant sur les personnes vivant avec le VIH. Enfin, une comparaison entre les besoins de santé ${ }^{4}$ vis-à-vis du $\mathrm{VIH} /$ sida des femmes immigrées $^{5}$ et non immigrées dans les deux enquêtes (Anrs-Vespa et Aides) sera réalisée.

\footnotetext{
Enquêtes disponibles auprès de l'association Aides

4 Le terme santé est entendu au sens que lui donne l'OMS et tel qu'il est repris dans la Charte d'Ottawa, à savoir la santé considérée comme une ressource pour les personnes et les groupes de population, ressource intégrant les dimensions sociales, psychologiques et culturelles de la vie des personnes.

5 Immigrées : femmes nées étrangères à l'étranger dont certaines ont pu acquérir par la suite la nationalité française suite à leur séjour sur le territoire français.
} 


\section{Méthodes}

Toutes les personnes participant à des activités régulières de Aides ont été sollicitées pour remplir le questionnaire. Pour celles qui ne souhaitaient pas le remplir, quelques données ont été recueillies, afin de pouvoir comparer les caractéristiques des personnes répondantes à celles des personnes non répondantes et d'estimer la " représentativité » de nos données.

Le recueil de données comportaitun auto-questionnaire contenant une centaine de questions sur les conditions de vie, la sexualité des répondants, les consommations de drogues, et des questions plus spécifiques au VIH et/ou aux hépatites. Les questions portant sur le VIH abordaient la révélation du statut sérologique à l'entourage, la prise d'un traitement antirétroviral et les effets secondaires de ces traitements. La perception des discriminations subies, l'origine et les circonstances de ces discriminations étaient également abordées. Le module sexualité renseignait sur les pratiques sexuelles des répondants. Les questions portaient à la fois sur les partenaires stables et occasionnels, la fréquence des rapports sexuels, l'utilisation ou non du préservatif, sur la possibilité d'aborder la question du VIH avant un rapport sexuel, sur la satisfaction de sa sexualité, sur le besoin et la possibilité d'en parler et avec qui.

\section{Résultats}

Au total, 2588 personnes ont été sollicitées. Parmi elles, 656 (25\%) ont refusé de répondre à l'enquête. La comparaison avec les 1932 personnes ayant accepté de répondre montre que les non-répondants étaient plus jeunes et plus souvent des personnes rencontrées en dehors des locaux de l'association [1].

\section{Les femmes accueillies à Aides}

Parmi les 1932 personnes ayant répondu à l'enquête, 633 (33\%) étaient des femmes, 1283 des hommes et 16 des transgenres. Quarante six pour cent ( $46 \%$ ) des femmes de l'échantillon ( $n=291), 46 \%$ des hommes et $25 \%$ des transgenres ont déclaré être séropositifs au $\mathrm{VIH} / \mathrm{sida}$.

Le tableau 1 montre la répartition des répondants à l'enquête, en fonction de leur sexe (femmes versus hommes + transgenres) et de leur statut sérologique par rapport au VIH. Le profil des femmes séropositives se distingue nettement des autres groupes sur plusieurs caractéristiques. La répartition par tranche d'âge des femmes séropositives est similaire à celle des hommes séropositifs, se caractérisant par une faible proportion de personnes de moins de 25 ans et une plus grande proportion de plus de 45 ans; on observe, parmi les femmes séropositives, une plus grande proportion d'immigrées $(38 \%)$. Plus souvent seules que les femmes séronégatives, elles ont en revanche plus souvent des enfants. Ce dernier résultat ne s'explique pas par une différence d'âge puisque le lien entre statut sérologique et avoir des enfants persiste même après ajustement sur l'âge. Le taux d'emploi est inférieur à celui des groupes non séropositifs, et elles perçoivent plus souvent l'allocation adulte handicapé. Cette précarité sociale ne s'accompagne pas en revanche d'une

Tableau 1

Caractéristiques des personnes ayant répondu à l'enquête * une semaine donnée, Aides 2005 *

\begin{tabular}{|c|c|c|c|c|c|c|}
\hline & $\begin{array}{c}\text { Total } \\
\mathrm{n}=1932\end{array}$ & $\begin{array}{l}\text { Femmes } \\
\text { séropositives } \\
\mathbf{n = 2 9 1}\end{array}$ & $\begin{array}{c}\text { Femmes } \\
\text { non séropositives } \\
\text { n }=342\end{array}$ & $\begin{array}{l}\text { Hommes + } \\
\text { Transgenres } \\
\text { séropositifs } \\
n=592\end{array}$ & $\begin{array}{c}\text { Hommes + } \\
\text { Transgenres } \\
\text { non séropositifs } \\
\mathrm{n}=707\end{array}$ & $p^{\star}$ \\
\hline & n (\%) & n (\%) & $\mathrm{n}(\%)$ & $\mathrm{n}(\%)$ & $\mathrm{n}(\%)$ & \\
\hline \multicolumn{7}{|l|}{ Âge } \\
\hline$<25$ ans & $321(16,6)$ & $11(3,8)$ & $137(40,1)$ & $13(2,2)$ & $160(22,6)$ & $* \star *$ \\
\hline $25-45$ ans & $1214(62,8)$ & $217(74,6)$ & $155(45,3)$ & $382(64,5)$ & $460(65,1)$ & \\
\hline$>45$ ans & $397(20,5)$ & $63(21,6)$ & $50(14,6)$ & $197(33,3)$ & $87(12,3)$ & \\
\hline Immigré(e) & $323(16,7)$ & $110(37,8)$ & $34(9,9)$ & $101(17,1)$ & $78(11,0)$ & $\star \star * *$ \\
\hline Vit en couple & $963(49,8)$ & $142(48,8)$ & $223(65,2)$ & $254(42,9)$ & $344(48,7)$ & $\star \star \star \star$ \\
\hline A des enfants & $608(31,5)$ & $176(60,5)$ & $110(32,2)$ & $168(28,4)$ & $154(21,8)$ & *** \\
\hline A un emploi ${ }^{1}$ & $620(32,1)$ & $62(21,3)$ & $99(28,9)$ & $141(23,8)$ & $318(45,0)$ & $* \star \star *$ \\
\hline Perçoit l'allocation adulte handicapé & $380(19,7)$ & $122(41,9)$ & $10(2,9)$ & $216(36,5)$ & $32(4,5)$ & $* * *$ \\
\hline Ne perçoit aucun revenu & $186(9,6)$ & $29(10,0)$ & $60(17,5)$ & $37(6,3)$ & $60(8,5)$ & $* * \star$ \\
\hline$A$ un logement stable ${ }^{2}$ & $1365(70,7)$ & $208(71,5)$ & $246(71,9)$ & $445(75,2)$ & $466(65,9)$ & ** \\
\hline Bénéficie d'une couverture maladie & $1384(94,9)$ & $287(98,6)$ & $318(93,0)$ & $566(95,6)$ & $663(93,8)$ & $* \star$ \\
\hline Usager(ère) de drogue & $384(19,9)$ & $23(7,9)$ & $68(19,9)$ & $59(10,0)$ & $234(33,1)$ & $* * *$ \\
\hline Homo/bisexuel(le) & $734(38,0)$ & $15(5,2)$ & $46(13,5)$ & $335(56,6)$ & $338(47,8)$ & $\star \star \star \star$ \\
\hline Séropositif(ve) pour le VHC & $317(16,4)$ & $62(21,3)$ & $39(11,4)$ & $119(20,1)$ & $97(13,7)$ & $\star \star \star$ \\
\hline
\end{tabular}

1 Temps plein ou partiel.

${ }^{2}$ Locataire ou propriétaire de son logement.

- Comparaison entre les différents groupes; ${ }^{* *} p<0,01 ;{ }^{* * *} p<0,001$ 
précarité plus importante vis-à-vis du logement. Le taux de séropositivité au VHC est plus élevé chez les femmes séropositives au VIH (21 \% des femmes séropositives sont co-infectées versus $11 \%$ des femmes séronégatives au VIH) et elles se déclarent moins souvent usagères de drogues et/ou homo/bisexuelles. Cette différence en fonction du statut sérologique se retrouve chez les hommes et chez les transgenres.

II apparaît qu'une part importante des femmes séropositives en contact avec l'association Aides, sont immigrées et en situation d'isolement affectif et de précarité économique. Les femmes non séropositives sont plus jeunes et sont plus souvent concernées par la consommation de produits psycho-actifs.

Ces résultats sont similaires à ceux observés dans les enquêtes précédentes, de même méthodologie, conduites par Aides en 2002 et 2004. Les femmes représentaient respectivement $29 \%$ et $34 \%$ de l'échantillon.

\section{Les femmes séropositives}

Parmi les 291 femmes séropositives incluses dans l'enquête, $38 \%$ sont de nationalité étrangère. Les trois-quarts de ces femmes sont originaires d'Afrique subsaharienne avec comme principaux pays d'origine le Cameroun ( $25 \%$ de l'échantillon), la Côte d'Ivoire (16\%) et le Congo (13\%). Six pour cent $(6 \%)$ sont centrafricaines, $4 \%$ maliennes, $4 \%$ tchadiennes ou togolaises et $3 \%$ burundaises, les autres nationalités d'Afrique subsaharienne représentant moins de $3 \%$ des effectifs. En dehors de l'Afrique subsaharienne, $4 \%$ sont originaires du continent américain, $2 \%$ du Maghreb, $3 \%$ d'Europe et $1 \%$ d'Asie. La date d'arrivée en France de ces femmes est relativement récente, puisque seulement $10 \%$ d'entre elles sont arrivées avant 1990 et la moitié depuis 2002. Elles sont $80,6 \%$ à avoir un permis de séjour. En revanche, la durée de ce permis est de un an seulement pour $60,2 \%$ d'entre elles et $26,7 \%$ seulement ont un permis d'une durée de plus d'un an. Elles sont également $77,6 \%$ à avoir une autorisation de travailler en France. La comparaison des caractéristiques sociodémographiques des femmes séropositives de nationalité française et des femmes séropositives immigrées montre que les difficultés rencontrées par ces femmes par rapport aux autres personnes de l'enquête sont encore plus accentuées parmi les immigrées : elles souffrent plus souvent d'un isolement affectif $(41,8 \%$ des femmes immigrées vivent en couple contre $53,0 \%$ des femmes de nationalité française, $p=0,063$ ), elles ont plus souvent des enfants $(72,7 \%$ versus $53,0 \%$ respectivement, $p=0,001$ ) et, si le taux d'emploi ne varie pas d'un groupe à l'autre $(25,5 \%$ versus $18,8 \%, p=0,178)$, les immigrées perçoivent moins souvent l'allocation adulte handicapé ( $33,6 \%$ contre $47,0 \%, p=0,026)$ et se déclarent plus souvent sans aucun revenu ( $23,6 \%$ versus $1,7 \%, p<0,001)$. L'usage de drogue concerne peu de femmes séropositives $(n=23$ ) et cellesci sont toutes de nationalité française.

\section{Les caractéristiques liées au VIH des femmes immigrées et non immigrées}

\section{Annonce de la séropositivité à l'entourage}

La positivité a été annoncée à certains de leurs amis par $63 \%$ des femmes, $62 \%$ l'ont annoncée à des membres de leur famille autres que le père et la mère et $56 \%$ à leur partenaire stable. Seules $39 \%$ et $31 \%$ l'ont annoncé respectivement à leur mère et à leur père. Dans tous les cas, les immigrées ont significativement moins souvent parlé de leur séropositivité que les femmes de nationalité française.

\section{Accès au dépistage et aux traitements}

Plus de $2 / 3$ des femmes immigrées ont découvert leur séropositivité depuis 2001 , contre $12 \%$ des femmes françaises. La proportion de femmes ayant un suivi médical pour leur infection par le VIH est de $95 \%$, celle des femmes séropositives prenant un traitement antirétroviral est de $77 \%$. Ces pourcentages ne diffèrent pas entre les deux groupes.

Les immigrées connaissent moins souvent leur taux de CD4 et leur niveau de charge virale que les femmes de nationalité française : $65,5 \%$ vs $77,3 \%(p<0,001)$ connaissent leur taux de CD4 et $14,9 \%$ versus $8,3 \%(p<0,001)$ connaissent leur charge virale. Les immigrées sont également traitées depuis moins longtemps que les femmes de nationalité française qui sont plus de la moitié à prendre un traitement depuis au moins cinq ans, alors que ce n'est le cas que pour $11,8 \%$ des femmes immigrées $(p<0,001)$.

La majorité des femmes $(60 \%)$ déclare ne pas avoir de difficulté à suivre le traitement. Près de la moitié juge le traitement anti-VIH très efficace, $22 \%$ en perçoivent les effets indésirables comme très gênants. Aucune différence n'est notée entre les deux groupes de femmes par rapport à ces deux items.

En revanche, Iorsqu'on explore les effets indésirables de manière plus précise, les femmes françaises déclarent significativement davantage de baisse de libido, de troubles du sommeil, de lipodystrophies, de diarrhées et de douleurs abdominales. De même, elles déclarent significativement plus souvent se faire aider sur le plan psychologique que les femmes immigrées. Ces différences dans les perceptions des effets indésirables peuvent résulter d'une différence dans la durée du traitement : dans certains cas, les effets indésirables apparaissent au long cours et sont moins marqués en début de traitement, dans d'autres cas, les personnes souffrent de devoir supporter les effets indésirables sur la durée [4]. Cette différence constatée peut aussi être rapprochée des témoignages [5] de personnes immigrées originaires d'Afrique subsaharienne pour lesquelles l'accès au traitement est perçu dans un contexte de survie biomédicale où la qualité de vie et les effets indésirables restent au départ très secondaires. 


\section{Les discriminations ressenties par les femmes séropositives}

Les femmes séropositives sont $54 \%$ à rapporter des discriminations, qui sont surtout liées à la séropositivité au VIH (79 \%) et qui concernent autant les immigrées que les femmes françaises (tableau 2). En revanche, $36 \%$ des femmes immigrées déclarent être discriminées du fait de leur origine géographique. Ces discriminations ont eu lieu pour $46 \%$ de l'ensemble des femmes séropositives dans le cadre de relations intimes et dans $39 \%$ des cas dans la vie quotidienne. Les femmes de nationalité française se sentent plus discriminées lors de soins, dans le travail ou les relations sexuelles, alors que les immigrées sont $43 \%$ à déclarer des discriminations dans leur communauté d'origine.

\section{Comparaison des femmes séropositives accueillies par Aides et des femmes séropositives de l'enquête Anrs-Vespa}

\section{Les difficultés sociales des femmes séropositives}

Les femmes constituent $29 \%$ des personnes incluses dans l'enquête Vespa (file active hospitalière). Parmi elles, une proportion similaire à celle de l'enquête de Aides est de nationalité étrangère $(36 \%)$. L'origine des femmes immigrées de Vespa est plus hétérogène que dans l'enquête de Aides, avec moins de femmes originaires d'Afrique sub-saharienne $(66,9 \%)$, une proportion plus importante de femmes originaires du Maghreb $(11,3 \%)$ et $10,1 \%$ originaires de pays européens.

Mis à part le Cameroun, qui représente le pays d'origine de $13 \%$ des femmes immigrées de Vespa, les pays africains sont présents dans des proportions similaires à celles de l'enquête de Aides : Côte d'Ivoire (14,2\%), Congo (11,8\%), Centrafrique $(5,8 \%)$, Tchad $(1,3 \%)$, Mali $(2,0 \%)$.

En revanche, les femmes immigrées de l'enquête Vespa sont arrivées en France depuis plus longtemps que celles qui fréquentent Aides. En effet, la moitié d'entre elles sont arrivées avant 1997 et seulement $10 \%$ sont en France depuis 2001. Plus des troisquarts $(77,1 \%)$ ont un permis de séjour avec, dans la moitié des cas, une durée de validité de dix ans ; $95 \%$ de ces femmes ont également un permis de travail.

Le tableau 3 montre la comparaison des caractéristiques sociodémographiques des deux échantillons, en fonction de la nationalité (française ou étrangère) des participantes.

Les répartitions par âge ne varient pas d'un échantillon à l'autre. Les femmes en contact avec l'association Aides souffrent en revanche d'un plus grand isolement affectif et social. Elles sont plus nombreuses à être seules, sans emploi, à percevoir l'allocation adulte handicapé, quelle que soit leur nationalité. Chez les femmes immigrées, les différences sont accentuées, notamment dans l'isolement, et se retrouvent également dans l'instabilité du logement et l'absence de ressources financières.

Tableau 2

Description des femmes séropositives victimes de discriminations (enquête " une semaine donnée Aides 2005 ")

\begin{tabular}{|c|c|c|c|c|}
\hline & $\begin{array}{c}\text { Total } \\
\mathrm{n}=157\end{array}$ & $\begin{array}{c}\text { Femmes } \\
\text { de nationalité française } \\
n=104\end{array}$ & $\begin{array}{c}\text { Femmes } \\
\text { immigrées } \\
n=53\end{array}$ & $\mathbf{p}^{\star}$ \\
\hline & $\mathrm{n}(\%)$ & $\mathbf{n}(\%)$ & $\mathbf{n}(\%)$ & \\
\hline \multicolumn{5}{|l|}{ Causes de la discrimination } \\
\hline Infection par le VIH & $124(79,0)$ & $83(79,8)$ & $41(77,4)$ & - \\
\hline Infection par le virus des hépatites $\mathrm{B}$ ou $\mathrm{C}$ & $19(12,1)$ & $16(15,4)$ & $3(5,7)$ & - \\
\hline Nationalité & $26(16,6)$ & $7(6,7)$ & $19(35,8)$ & 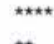 \\
\hline Consommation de drogues & $16(10,2)$ & $15(14,4)$ & $1(1,9)$ & $*$ \\
\hline \multicolumn{5}{|l|}{ Lieux de la discrimination } \\
\hline Le travail & $35(22,3)$ & $30(28,8)$ & $5(9,4)$ & $\star \star \star \star$ \\
\hline Les soins de santé & $48(30,6)$ & $41(39,4)$ & $7(13,2)$ & 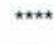 \\
\hline
\end{tabular}

${ }^{1}$ Amis, conjoint, famille.

2 Personnes d'origine étrangère, communauté gay etc.

* $p$ : comparaison entre les différents groupes.

$-p$ non significatif ; ${ }^{* *} p<0,05 ;{ }^{* * *} p<0,01 ;{ }^{\cdots * *} p<0,001$. 
Tableau 3

Comparaison des femmes de l'enquête Vespa et des femmes séropositives de l'enquête * une semaine donnée Aides 2005 "

\begin{tabular}{|c|c|c|c|c|c|c|}
\hline & $\begin{array}{c}\text { Femmes } \\
\text { de nationalité } \\
\text { française de Vespa }\end{array}$ & $\begin{array}{c}\text { Femmes } \\
\text { de nationalité } \\
\text { française de EUSD } \\
\text { Aides }\end{array}$ & $\mathbf{p}$ & $\begin{array}{c}\text { Femmes } \\
\text { immigrantes } \\
\text { de Vespa }\end{array}$ & $\begin{array}{c}\text { Femmes } \\
\text { immigrantes } \\
\text { de EUSD Aides }\end{array}$ & $\mathbf{p}^{\star}$ \\
\hline & $\mathrm{n}=542$ & $n=181$ & & $n=303$ & $n=110$ & \\
\hline & $\mathrm{n}(\%)$ & $\mathrm{n}(\%)$ & & n (\%) & $\mathrm{n}(\%)$ & \\
\hline \multicolumn{7}{|l|}{ Âge } \\
\hline$<25$ ans & $20(3,6)$ & $4(2,2)$ & - & $10(3,5)$ & $7(6,4)$ & - \\
\hline $25-45$ ans & $380(70,1)$ & $133(73,5)$ & & $242(79,8)$ & $84(76,4)$ & \\
\hline$>45$ ans & $143(26,3)$ & $44(24,3)$ & & $51(16,7)$ & $19(17,3)$ & \\
\hline Vit en couple & $339(62,6)$ & $96(53,0)$ & ** & $179(58,9)$ & $46(41,8)$ & $* * *$ \\
\hline A des enfants & $308(56,7)$ & $96(53,0)$ & - & $234(77,1)$ & $80(72,7)$ & - \\
\hline A un emploi ${ }^{1}$ & $238(43,8)$ & $34(18,8)$ & 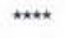 & $132(43,3)$ & $28(25,5)$ & $\cdots$ \\
\hline Perçoit l'allocation adulte handicapé & $147(27,1)$ & $85(47,0)$ & $* * * \star$ & $42(13,9)$ & $37(33,6)$ & $* * * *$ \\
\hline Ne perçoit aucun revenu & $24(4,3)$ & $3(1,7)$ & - & $6(1,9)$ & $26(23,6)$ & 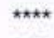 \\
\hline A un logement stable ${ }^{2}$ & $493(91,0)$ & $163(90,1)$ & - & $200(66,0)$ & $45(40,9)$ & $\star * * *$ \\
\hline
\end{tabular}

1 Temps plein ou partiel.

${ }^{2}$ Locataire ou propriétaire de son logement.

* $p$ : comparaison entre les différents groupes.

$-p$ non significatif ; ${ }^{* *} p<0,05 ;{ }^{\star * *} p<0,01 ;{ }^{\cdots * *} p<0,001$.

\section{La révélation de la séropositivité}

Si les taux de révélation au père et/ou à la mère sont très faibles et sensiblement les mêmes chez les immigrées dans les deux enquêtes (respectivement 9,1 \% et $12,7 \%$ dans l'enquête Aides versus $8,3 \%$ et $14,2 \%$ dans l'enquête Vespa), en revanche, chez les femmes de nationalité française, les taux sont beaucoup plus bas dans l'enquête Vespa que dans l'enquête Aides (23,2 \% versus $44,8 \%$ pour le père ; $36,9 \%$ versus $54,7 \%$ pour la mère). La proportion de révélation aux amis est là encore inférieure dans l'enquête Vespa, que ce soit chez les femmes françaises (Vespa $43,4 \%$ / Aides $75,7 \%$ ) ou chez les femmes immigrées (Vespa $15,8 \%$ / Aides $42,7 \%$ ).

Pour ce qui concerne les discriminations, les comparaisons sont rendues difficiles car cet aspect est abordé de manière plus sommaire dans Vespa. Les questions ne sont relatives qu'aux discriminations ressenties du fait de la séropositivité. Des discriminations de la part de la famille sont rapportées par 16,6\% des femmes françaises et par $9,2 \%$ des femmes immigrées $(p<0,001)$. Les discriminations de la part des amis sont rapportées par $17 \%$ des femmes françaises et $7,3 \%$ des immigrées $(p<0,001)$, et celles de la part de partenaires sexuels respectivement par $21,4 \%$ et $7,9 \%(p<0,001)$.

Les différences perçues et déclarées dans les discriminations entre les femmes immigrées et françaises doivent être interprétées avec prudence, un groupe pouvant être plus sensible socialement à un type de discrimination ou attribuer la discrimination préférentiellement à une cause plutôt qu'à une autre, notamment la couleur de la peau et/ou l'infection par le VIH.

Les femmes en contact avec Aides ont plus souvent révélé leur séropositivité à leur entourage que celles incluses dans l'enquête
Vespa. La révélation du statut expose à subir en retour des discriminations, ce qui est confirmé dans notre enquête. Ces résultats pourraient s'expliquer soit par le fait que les femmes ayant fait la démarche d'entrer en contact avec une association de lutte contre le sida y ont trouvé un support pour dire leur séropositivité à leur entourage, soit au contraire parce qu'elles ont été victimes de discriminations liées au dévoilement de leur séropositivité.

\section{Sexualité des femmes séropositives et prises de risque}

\section{Activité sexuelle}

L'activité sexuelle est peu élevée ( $48 \%$ ) pour une population dont $78 \%$ des personnes ont moins de 45 ans (tableau 4). Par ailleurs, $48 \%$ des femmes déclarent une relation stable, ce chiffre étant plus bas chez les immigrées. Parmi l'ensemble des femmes séropositives, 93 (soit $66 \%$ ) sont dans un couple sérodifférent ${ }^{6}$ stable, sans qu'il y ait de différence entre les femmes immigrées et les autres. Pourtant, pour $30 \%$ des immigrées, le statut sérologique du partenaire est inconnu, alors que ce n'est le cas que pour $7 \%$ des femmes de nationalité française. La révélation du statut sérologique du partenaire apparaît plus problématique pour les immigrées que pour les autres femmes.

Par ailleurs, $13 \%$ des femmes déclarent des partenaires occasionnels dans les trois derniers mois (tableau 4), sans qu'aucune différence ne soit observée entre les deux groupes. Concernant la connaissance du traitement d'urgence (prophylaxie post-

6 Dans l'enquête, le partenaire d'une femme sẻropositive est dit sérodifférent sì il est séronégatif ou de statut sérologique non connu de la femme du couple. 
Tableau 4

Sexualité des femmes séropositives ayant répondu à l'enquête « une semaine donnée Aides 2005 "

\begin{tabular}{|c|c|c|c|c|}
\hline & $\begin{array}{l}\text { Total } \\
\mathrm{n}=\mathbf{2 9 1}\end{array}$ & $\begin{array}{c}\text { Femmes de } \\
\text { nationalité française } \\
n=181\end{array}$ & $\begin{array}{l}\begin{array}{c}\text { Femmes } \\
\text { immigrées }\end{array} \\
n=110\end{array}$ & $\mathbf{p}^{\star}$ \\
\hline & $\mathrm{n}(\%)$ & $\mathbf{n}(\%)$ & $\mathrm{n}(\%)$ & \\
\hline \multicolumn{5}{|c|}{ Fréquence des rapports sexuels au cours des 4 dernières semaines } \\
\hline 0 & $151(51,9)$ & $89(49,2)$ & $62(56,4)$ & $* *$ \\
\hline 1 à 4 & $71(24,4)$ & $40(22,1)$ & $31(28,2)$ & \\
\hline 5 à 8 & $29(10,0)$ & $22(12,2)$ & $7(6,4)$ & \\
\hline Plus de 9 & $22(7,6)$ & $19(10,5)$ & $3(2,7)$ & \\
\hline Non-réponse & $18(6,2)$ & $11(6,1)$ & $7(6,4)$ & \\
\hline Pratique de la pénétration les 3 derniers mois & $121(41,6)$ & $79(43,6)$ & $42(38,2)$ & - \\
\hline Relation stable & $140(48,1)$ & $96(53,0)$ & $44(40,0)$ & $*$ \\
\hline Partenaires occasionnels les 3 derniers mois & $38(13,1)$ & $25(13,8)$ & $13(11,8)$ & - \\
\hline Connaissance du traitement d'urgence & & & & *** \\
\hline Oui & $149(51,2)$ & $113(62,4)$ & $36(32,7)$ & \\
\hline Non & $103(35,4)$ & $44(24,3)$ & $59(53,6)$ & \\
\hline Non-réponse & $39(13,4)$ & $24(13,3)$ & $15(13,6)$ & \\
\hline \multicolumn{5}{|l|}{ Satisfaction vis-à-vis de sa sexualité } \\
\hline Satisfait/plutôt satisfait & $128(44,0)$ & $78(43,1)$ & $50(45,5)$ & ** \\
\hline Plutôt insatisfait/insatisfait & $121(41,6)$ & $83(45,9)$ & $38(34,5)$ & \\
\hline Non-réponse & $42(14,4)$ & $20(11,0)$ & $22(20,0)$ & \\
\hline \multicolumn{5}{|l|}{ Besoin de parler de sa sexualité } \\
\hline Souvent & $128(44,0)$ & $17(9,4)$ & $14(12,7)$ & - \\
\hline Parfois & $114(39,2)$ & $78(43,1)$ & $36(32,7)$ & \\
\hline Rarement & $71(24,4)$ & $47(26,0)$ & $24(21,8)$ & \\
\hline Jamais & $51(17,5)$ & $28(15,5)$ & $23(20,9)$ & \\
\hline Non-réponse & $24(8,2)$ & $11(6,1)$ & $13(11,8)$ & \\
\hline Possibilité de parler de sa sexualité & & & & * \\
\hline Souvent & $37(12,7)$ & $19(10,5)$ & $18(16,4)$ & \\
\hline Parfois & $98(33,7)$ & $58(32,0)$ & $40(36,4)$ & \\
\hline Rarement & $93(32,0)$ & $65(35,9)$ & $28(25,5)$ & \\
\hline Jamais & $41(14,1)$ & $30(16,6)$ & $11(10,0)$ & \\
\hline Non-réponse & $22(7,6)$ & $9(5,0)$ & $13(11,8)$ & \\
\hline \multicolumn{5}{|l|}{ Possibilité de parler de sa sexualité avec } \\
\hline Un médecin pour le VIH & $116(39,9)$ & $69(38,1)$ & $47(42,7)$ & - \\
\hline Un médecin généraliste & $59(20,3)$ & $41(22,7)$ & $18(16,4)$ & - \\
\hline Un spécialiste de la sexualité (sexologue) & $20(6,9)$ & $12(6,6)$ & $8(7,3)$ & - \\
\hline Un psychologue ou un psychiatre & $62(21,3)$ & $47(26,0)$ & $15(13,6)$ & ** \\
\hline Dans des actions de Aides & $101(34,7)$ & $60(33,1)$ & $41(37,3)$ & - \\
\hline Avec des amis ou des proches & $133(45,7)$ & $91(50,3)$ & $42(38,2)$ & ** \\
\hline
\end{tabular}

- Comparaison entre les différents groupes.

- $p$ non significatif ; ${ }^{* *} p<0,05 ; \cdots p<0,001$.

exposition), les femmes immigrées sont largement défavorisées, puisque seules $33 \%$ d'entre elles savent qu'un tel traitement existe, contre $62 \%$ des femmes françaises (tableau 4). À noter que cet écart de connaissance sur le traitement d'urgence est aussi observé parmi les personnes incluses dans Vespa.

Pour ce qui concerne la satisfaction ou l'envie de parler de sa sexualité, les deux groupes de femmes ne sont pas différents. La moitié d'entre elles déclare être satisfaite de sa sexualité. En revanche, les femmes immigrées ont plus souvent l'occasion d'en parler (tableau 4). Les femmes de nationalité française parlent majoritairement de leur sexualité avec leurs amis (une femme sur deux), $38 \%$ en parlent avec leur médecin pour le VIH et $33 \%$ dans les actions de Aides. Les femmes immigrées ont, quant à elles, comme interlocuteur privilégié le médecin qui les suit pour leur infection par le VIH ( $43 \%$ ), puis viennent les amis et les actions de Aides (38\% et $37 \%$ respectivement) (tableau 4). 
Si le taux d'activité sexuelle des femmes séropositives de l'enquête Aides est sensiblement le même que celui des femmes incluses dans Vespa, ces dernières déclarent un plus grand nombre de rapports sexuels au cours des quatre semaines précédant l'enquête. Comme cela a déjà été mentionné, les femmes en contact avec Aides sont plus souvent seules, en particulier celles de nationalité française, et elles déclarent moins de partenaires occasionnels. Le taux d'insatisfaction vis-à-vis de la sexualité rapporté par ces femmes vient renforcer ce constat d'isolement affectif et relationnel.

\section{Sexualité et prise de risque}

\section{Relation avec un partenaire stable sérodifférent}

On a défini comme sérodifférent un couple composé d'une femme séropositive et d'un partenaire séronégatif ou de statut inconnu. La prise de risque est définie comme la déclaration d'un ou de plusieurs rapports sexuels non protégés avec ce partenaire, au cours des trois derniers mois.

Une prise de risque sexuel avec un partenaire stable sérodifférent est déclarée par $20 \%$ des femmes ayant un partenaire sérodifférent. Elle ne varie pas entre femmes françaises et immigrées.

On peut cependant noter que les immigrées parlent davantage de leur situation par rapport au VIH avec leur partenaire stable que les autres femmes $(20,7 \%$ versus $5,0 \%, p=0,03)$. A l'inverse, nous avons souligné précédemment que le statut du partenaire était moins souvent connu chez les immigrées que chez les femmes françaises.

\section{Relations avec des partenaires occasionnels}

Pour ce qui concerne les relations avec des partenaires occasionnels, peu de différences apparaissent, et ce d'autant plus que seulement 38 femmes séropositives disent en avoir. Parmi ces dernières, $55 \%$ déclarent entre un et cinq partenaires occasionnels dans les trois derniers mois et parmi ces femmes, $24 \%$ déclarent ne pas utiliser systématiquement un préservatif lors des pénétrations. La faiblesse des effectifs ne permet pas d'interpréter ce qui sous-tend cette conduite. On peut noter toutefois que le fait de parler de sa situation par rapport au VIH avant des rapports sexuels avec un partenaire occasionnel ne concerne qu'un tiers des femmes qui répondent à cette question.

\section{La place de l'enquête " Aides et toi " dans la prise en compte des besoins de santé des femmes accueillies à Aides}

Les femmes séropositives ont un contact très fréquent avec l'association, au moins une fois par mois pour plus de $70 \%$ d'entre elles. Cela n'est le cas que de $61 \%$ des hommes et de $47 \%$ des femmes non séropositives ${ }^{7}$. Un tiers de ces dernières ont rempli le questionnaire d'enquête lors de leur premier contact avec l'association.

Public marqué par une forte précarité sociale et financière, plus de la moitié des femmes séropositives ont fait appel au soutien associatif dans les démarches sociales et aux aides financières proposés dans les délégations départementales de Aides. Les autres actions auxquelles elles ont activement participé sont les permanences d'accueil ( $40 \%$ ), le soutien par la convivialité et/ou les loisirs ( $38 \%)$, les groupes de parole et d'entraide $(23 \%)$ et les actions spécifiquement réservées aux femmes (18\%).

La prise en compte des besoins de santé des femmes accueillies et, plus spécifiquement, des femmes séropositives au VIH, passe par une approche globale de la santé qui ne saurait être réduite au soin ou à une approche médicale stricto sensu. Elle conjugue un soutien d'ordre médicosocial (revenus, logement, ...), une approche plus cognitive d'accompagnement à l'appropriation des informations (modes de transmission, traitements) et un soutien par les pairs (vivre avec le virus dans les différentes dimensions du quotidien, notamment la vie affective et sexuelle). La participation des femmes à ces actions et les besoins pris en compte sont peu visibles et peu accessibles à ce type d'enquête, en partie parce que les actions de santé animées par Aides et destinées aux personnes séropositives et/ou atteintes par les hépatites virales sont, de manière générale, ouvertes à l'ensemble des populations concernées par ces épidémies. Les principes d'action communautaire autour du VIH (et non identitaire sur le genre ou l'orientation sexuelle) qui guident l'association favorisent un " mélange » de personnes de genres, d'origines, d'orientations sexuelles, de parcours différents.

Ces principes d'action et la volonté de ne pas imposer aux personnes des questionnements pouvant évoquer, au moins symboliquement, sinon des discriminations, du moins des ségrégations, ont souvent conduit à ne pas chercher à identifier des " catégories " telles que femmes, hommes, transgenre, immigré ou autochtone etc.

\section{Conclusion}

L'enquête "Aides et toi 2005 " montre que le groupe des femmes séropositives cumule des facteurs défavorables en ce qui concerne la qualité de vie, la capacité à se soigner et le développement de la capacité de prévention. Ces facteurs tiennent aux conditions de vie de ces femmes, souvent isolées et en grande précarité sociale, économique et relationnelle. Ils sont plus marqués pour les femmes accueillies à Aides que pour la population générale des femmes séropositives suivies à l'hôpital et étudiée par l'enquête Vespa.

\footnotetext{
Non séropositive : femme déclarant être séronégative ou ne connaissant pas ou plus son statut sérologique par rapport au VIH (séro-interrogative)
} 
Parmi les femmes séropositives, les immigrées /étrangères sont plus fragilisées économiquement et socialement. De plus, elles ressentent davantage de discriminations, notamment dans leur communauté. Ce surcroît de difficultés, qui différencie les femmes des deux enquêtes, peut s'expliquer pour les femmes immigrées. La nature des titres de séjour (durée inférieure à un an) et l'absence d'autorisation de travail sont des indicateurs d'une arrivée récente en France, qui s'accompagne d'énormes difficultés d'accès au système d'aide sociale, lui-même conditionné à la durée de résidence sur le territoire français. L'isolement affectif et les discriminations intracommunautaires viennent alors s'ajouter pour rendre encore plus difficile l'accès, pour ces femmes fraîchement immigrées et souvent débutant un traitement, à un environnement favorable aux soins et à la prévention.

Dans ce contexte, l'intervention de Aides peut être considérée comme un lieu d'expression et de recueil des difficultés que connaissent les femmes les plus précarisées, notamment les femmes issues de l'immigration, dans le domaine social comme dans ceux de la vie affective, sexuelle et de la prévention. L'identification de ces difficultés peut favoriser, dans un cadre associatif comme de manière plus large, l'élaboration de réponses collectives en termes de soutien.

\section{Références bibliographiques}

1. Aides. Aides et toi Résultats 2005. Paris : 2006

2. Aides. Rapport d'activité 2005. Paris: DGS, 2005.

3. Lert F, Obadia $Y$ et al. First results from the Anrs-Vespa survey of persons living with HIV/AIDS. Rev Epidemiol Sante Publique $2005 ; 53(1)$ : 79-85.

4. Aides. Actes des États généraux des personnes vivant avec le VIH. Paris: 2004.

5. Aides. Vivre en France au temps du sida. RemAides, numéro spécial, 2005. 